\title{
FAST IMAGING APPLICATIONS IN THE NUCLEAR TEST PROGRAM
}

\author{
Richard Lear
}

This paper was prepared for submittal to

Nuclear Science Symposium, October 19-21, 1983,

Sheraton Paiace Hotel, San Francisco, CA

October 14, 1983

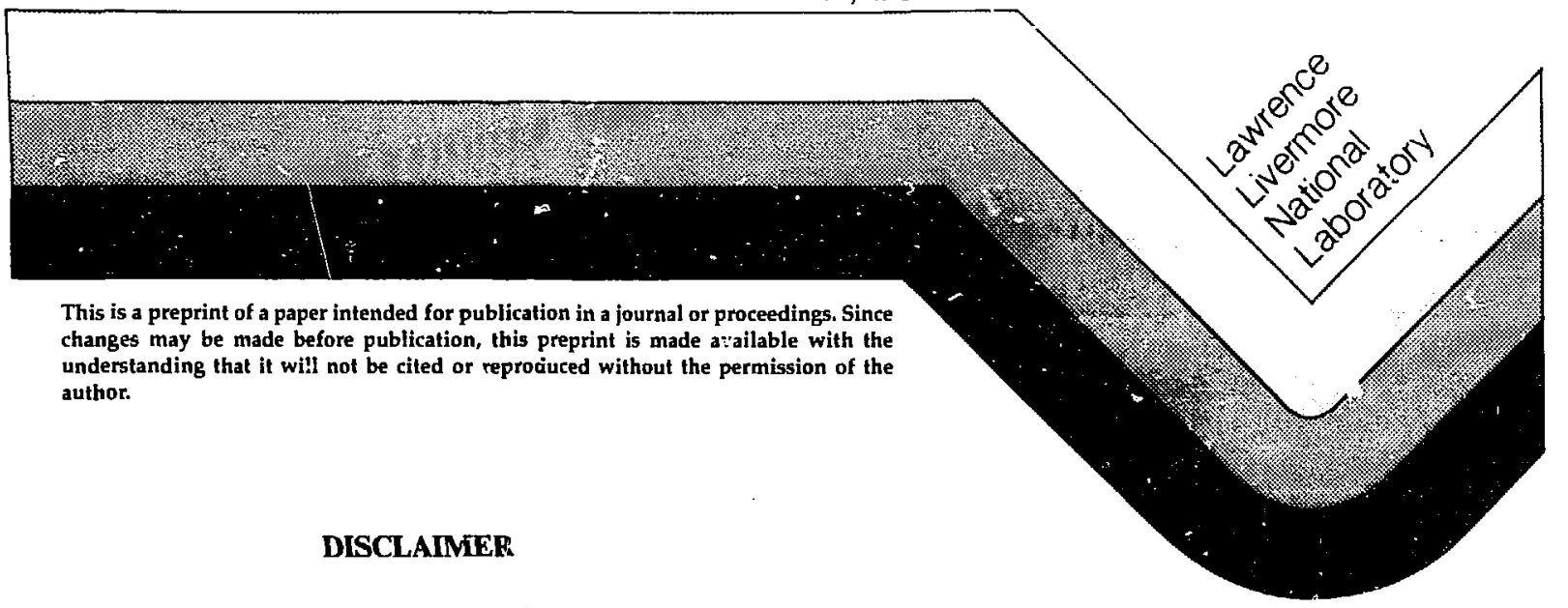

This report was prepared as an account of work sponsored by an agency of the United States Government. Neither the United States Government nor any agency thercof, nor any of their employees, makes any warranty, express or implied, or assumes any legal liability or responsibility for the accuracy, completeness, or usefulness of any information, apparatus, product, or process disclosed, or represents that its use would rot infringe privately owned rights. Reference herein to any specific commercial product, process, or service by trade name, trademark, manufacture, or otherwise does not necessarily constitute or imply its endorsement, recommendation; or favoring by the United States Government or any agency thereof. The views and opinions of authors expressed herein do not necessarily state or reflect those of the United States Government or any agency thereof. 


\title{
FAST IMAGING APPLICATIONS IN THE NUCLEAR TEST PROGRAM
}

\author{
Richard Lear \\ P.O. Box 808 \\ Lavirence Livermore National Laboratory \\ Livermore. California 94550
}

\section{Abstract}

Applications of fast imaging employ both streak cameras and fast framing techniques. Image intensifier tubes are gated to provide fast two-dimensional shutters of $2-3$ ns duration with shatter ratios of greater than $10^{6}$ and resolution greater than $10^{4}$ pixels. Shutters of less than I ns have been achicved with experimental tubes. Characterizition data demonstrate the importance of tube and pulser design.

Streak cameras are used to simultaneously record temporal and intensity information from up to 200 spatial points. Streak cameras are combined with remote readout for downhole uses and re coupled to fiber optic cables for uphole uses. Optical wavelength multiplexing is being studied as a means of compressing additional image data onto optical fibers. Performance data demonstrate trade-ofis betwcen image resolution: and system sensitivity.

\section{Ir:troduction}

Imaging is used in underground nuclear testing to study implosion symmetry and bum efficiency of fission or fusion devices. The inaging system is actually a very large pinhole camera, as shown in Fig. 1 . The size, shape, and symmetries of a radiation-emitting volume are measured by imaging the radiation thro'sgh a 20-in.-long stee! "pinhole" and onto a plastic fluorescer. The pinhole design is tiagrammed in Fig. 2. The image from the fluor is captured by unique. fast TV cameras, then digitized and transmitted uphole to be stored in computer memories. The digital data are combined with calibration information to produce pictures that show the si e, shape, and density distribution of fission or fusion materials. Imaging experiments can resolve $x$-ray, gamma ray, fission neutron. or fusion neutron images to $0.10 \mathrm{~mm}$. Images from the different 1 .'pes of radiation can be scparated by filtering to remove the undesired radiation species or by use of time-of-flight techniques in which cameras are shuttered on only

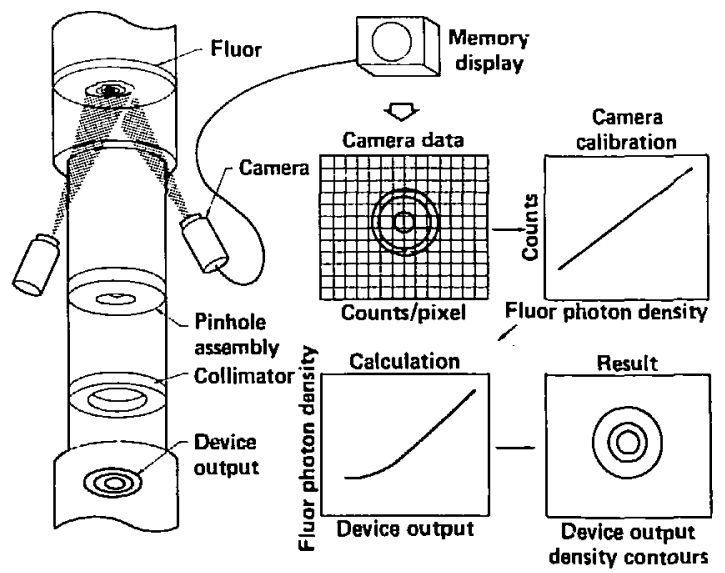

FIG. 1. PINEX (PINhole EXperiment) focused radiation frort a nuclear source through a "pinhole" and onto a plastic fluor. Light from the fluor is focused onto special high-speed TV cameras, which digitize the data downhole and send them uphole to be stored in a computer memory. The data are later combined with calibration data to produce a quantized image.

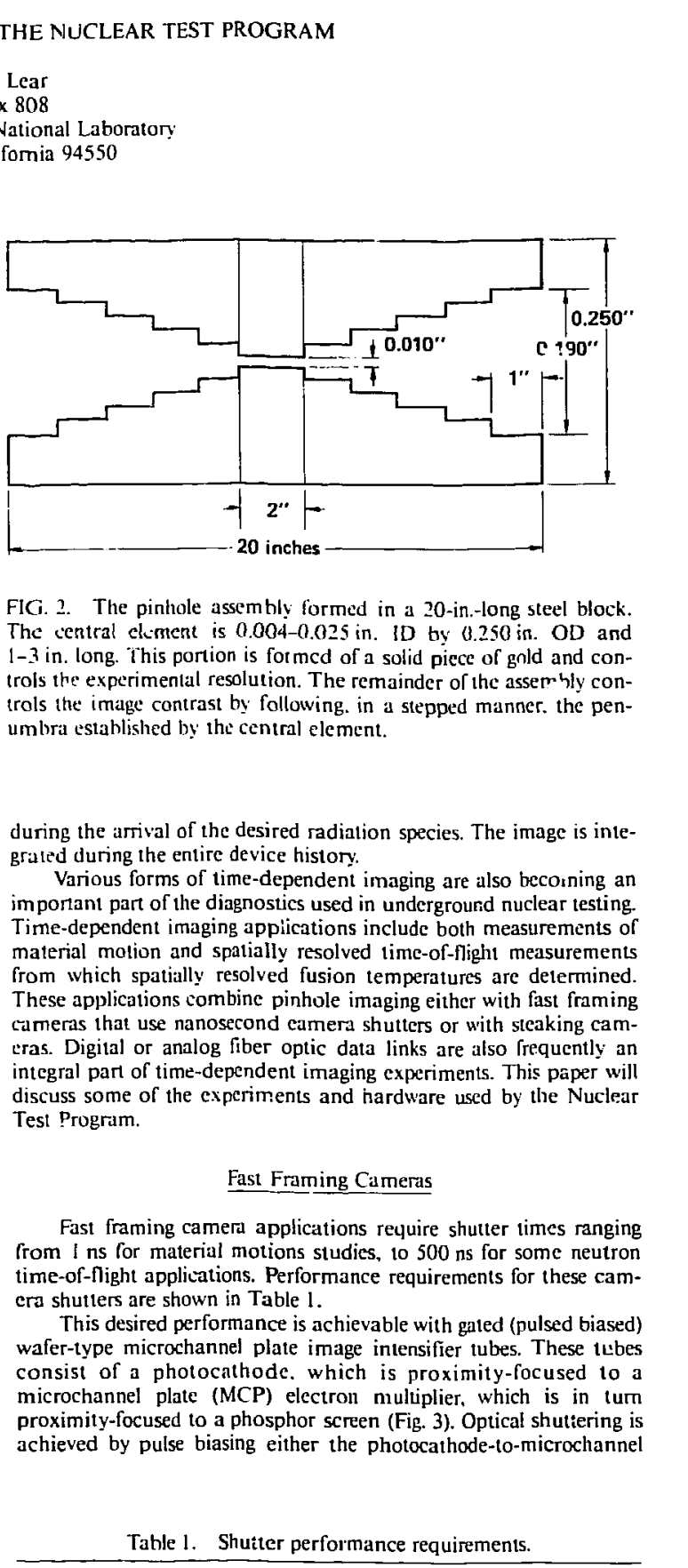

FIG. 2. The pintole assembly formed in a 20 -in.-long steel block. The central eliment is $0.004-0.025 \mathrm{in}$. ID by $0.250 \mathrm{in}$. $O D$ and $1-3$ in. long. This portion is for med of a solid piece of gold and controls the experimental resolution. The remainder of the assem bly controls the image contrast by following. in a stepped manner. the penumbra established by the central element.

during the arrival of the desired radiation species. The image is integraled during the entire device history.

Various forms of time-dependent imaging are also becosning an important part of the diagnostics used in underground nuclear testing. Time-dependent imaging applications include both measurements of material motion and spatially resolved time-of-flight measurements from which spatially resolved fusion temperatures are determined. These applications combine pinhole imaging either with fast framing cameras that use nanosecond eamera shutters or with steaking camcras. Digital or analog fiber optic data links are also frequently an integral part of time-dependent imaging experiments. This paper will discuss some of the expcriments and hardware used by the Nuclear Tesı Program.

\section{Fast Framing Cameras}

Fast framing camera applications require shutter times ranging from I ns for material motions studies, to $500 \mathrm{~ns}$ for some neutron time-of-flight applications. Performance requirements for these camera shutters are shown in Table 1.

This desired performance is achievable with gated (pulsed biased) wafer-type microchannel plate image intensifier tubes. These tebes consist of a photocathode. which is proximity-focused to a microchannel plate (MCP) electron multiplier, which is in tum proximity-focused to a phosphor screen (Fig. 3). Optical shutiering is achieved by pulse biasing either the photocathode-to-microchannel

Tahle l. Shuiter performance requirements.

\begin{tabular}{ll}
\hline Spatial rusolution & $>10^{4}$ pixels \\
Temporal resolution & $1 \mathrm{~ns}$ to $500 \mathrm{~ns}$ \\
Optical gain & $10^{3}$ \\
Shutter ratio & $10^{6}: 1$ \\
Dynamic range & $>200: 1$ linear \\
Response uniformity & No dynamic distortion \\
Spatial distortion & No dynamic distortion \\
\hline
\end{tabular}




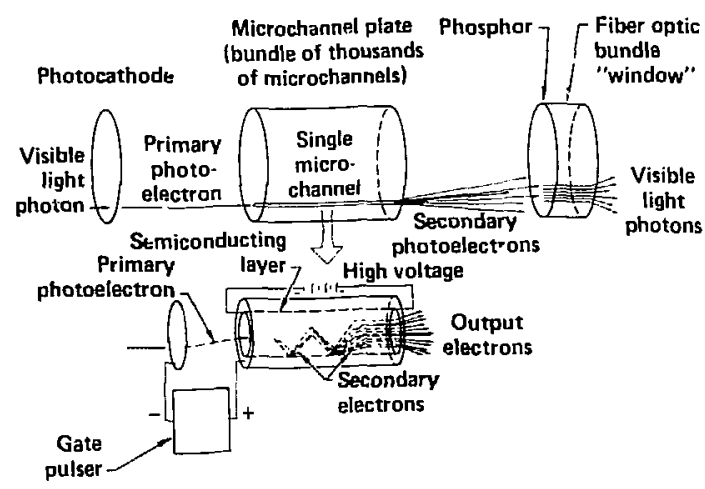

FIG. 3. Image intensifier tubes. The tube is gated by reverse biasing between the photocathor : and the microchannel plate, then supplying a pulsed forward bias voltage.

plate or the MCP itself. We have experimented with gating the photocathode in both a coaxial termination geometry and in a microstripline transmission geometry.

\section{Optical Shuter Chanclerization}

Figure 4 is a diagram of the laboratory we use to characterize these shutters. An Electro-Photonics Limited flashlamp pumped-dye laser is passively modelocked to provide a train of 100-200 laser pulses, each separated by $5 \mathrm{~ns}$. Each pulse has a duration of approkimately $15 \mathrm{ps}$ with an output wavelenglh centered at $600 \mathrm{~nm}$. The ratio of the selected pulse to a nonselected pulse is approximateiy $400: 1$. Part of the energy of the selected pulse is split off and used both to generate the trigger signal for the pulser driving the intensifier tube and to provide a monitor of the laser output to ensure proper laser performance. In this way the electrical pulser used to gate the tube is synchronized to the sclected laser pulse. The remainder of the laser pulse is sent through an optical delay before being directed to the photocathode of the intensifier tube. The laser pulse is walkca through the optical gate in steps of 100 ps by varving the length of the optical delay path. At each step, the output frum :he intensifier tube phosphor is recorded by a solid-state camera built azound the Reticon $100 \times 100$ array. The camera is fiber-opticilly coupied to the phosphor faceplate of the intensifier tube. The output of the camcra is digitized and sent to a Digital Equipment Corpcration LSl-il min:computer, where the recorded image is saved on disk. Imageprocessing routines are used to analyze the data and to deternine the performance parameters of the optical shutter.

When possible, the light level input to the tube is adjusted in produce at least 100 counts above background on the recording camera in order to achicve a system-dynamic range (maximum recordable signal/minimum recordable signal) of at least 100:1. The total oftical gate length is defined as the time from when the output from the intensifier tube can first be detected above the camera noise to when the intensifier tube output is down to a level that cannot be distinguished from the camera noise. ine tum-on time is defined as the time from detection of the output at the edge of the intensifier tube to the time when the output foom the center of the tube is the same intensity as the output at the edge. The turn-of time is defined as the time from the beginning of drop-off of the output at the edges of the intensifier tube to the time when the intensifier tube is completely oft.

Resolution is measured using a $100 \%$ contrast target in contact with the fiber-optic faceplate of the intensifier tube. To ensure uniform input, a milk glass diffuser is placed approximately 6 in. in front of the photocathode of the intensifier tube. A CTF (contrast transfer function) value is then read of the intensity profile of the digitized image recorfed by the Reticon camera.

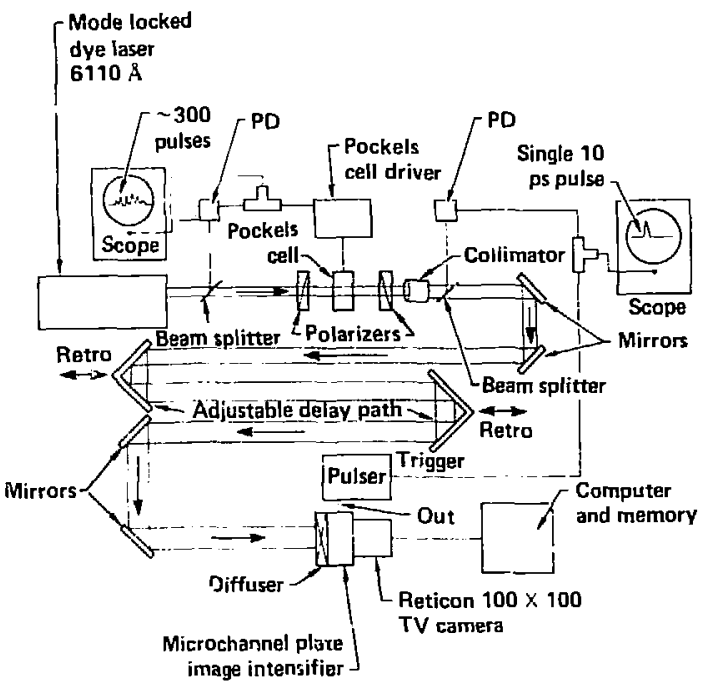

FIG. 4. Diagram of the characterization setup used to characterize the temporal and spatial performance of MCP intensifier tubes.

\section{Electrical Pulsers}

Fast electrical pulsers are nezded in order to quickly gate intensifi:d ubes. ${ }^{\prime}$ Applications involving $1-5$ ns optical gates require very low jitter. An ideal photocathode fulser would be capable of supplying $100-200 \mathrm{~V}$ into a low impedance (approximately 17 ohms) with a rise and fall time of $200-300 \mathrm{ps}$, would have a full width al half maximum (FWHM) of approximately 500 ps. and would exhibit 30-ps jitter.

Avalanche transistors. or avalanche transistors combined with step recovery diodes, are used to provide the electrical gatte pulse for photocathode gating. Voltage pulses of $200 \mathrm{~V}$ with a rise time of $1.1 \mathrm{~ns}$ can be gencrated by Ravitheon RS 3500 avalanche transistors in a charge-line pulse. Step-recovery diodas can be used to sharpen the rising and falling edges of the pulses. ${ }^{2} A$ pulser manufactured by Power Spectra, Ine. (formerly Picosecond Enterprises. Inc.) delivers $170 \mathrm{~V}$ with a rise and fall time of $500 \mathrm{ps}$ into a $17-\mathrm{ohm}$ microstrip transmission line. We have used especially packaged step-recovery diodes to deliver $200-\mathrm{V}$ pulses with a 300 -ps rise time and a 450 -ps fall time into a 50 -ohm load. We are coutinuing with further repackaging to reduce package capacitance and inductance and to provide a better impedance match between the diode package and the microstrip transmission line.

The photoconductive switch is another currently available technique to produce voitage pulses of several hundred volts or even kilovolts, with rise and fall times on the order of a few hundred picoseconds. ${ }^{3-5}$ However, a laser that is capable of providing several microjoules is needed for driving the switch. Such systems are not suited for field applications, where portability and reliability are essential, although they are useful for laboratory evaluations. Photoconductive switches may find future uses in the field if laser diodes can be made to drive them.

\section{Characterization Resuls}

Figure 5 shows the results of gating an $18-\mathrm{mm}$ intensifier tube that has a standard S20 photocathode. The picture in the upper left comer shows the electrical driving pulse; the other pictures show the intensity recorded along a diameter of the tube at various times during the optical gate. The photocathode was reverse biased to $+50 \mathrm{~V}$, and $\mathrm{a}-200 \mathrm{~V}$ pulse ivith an FWHM of $38 \mathrm{~ns}$ was applied in a termination 


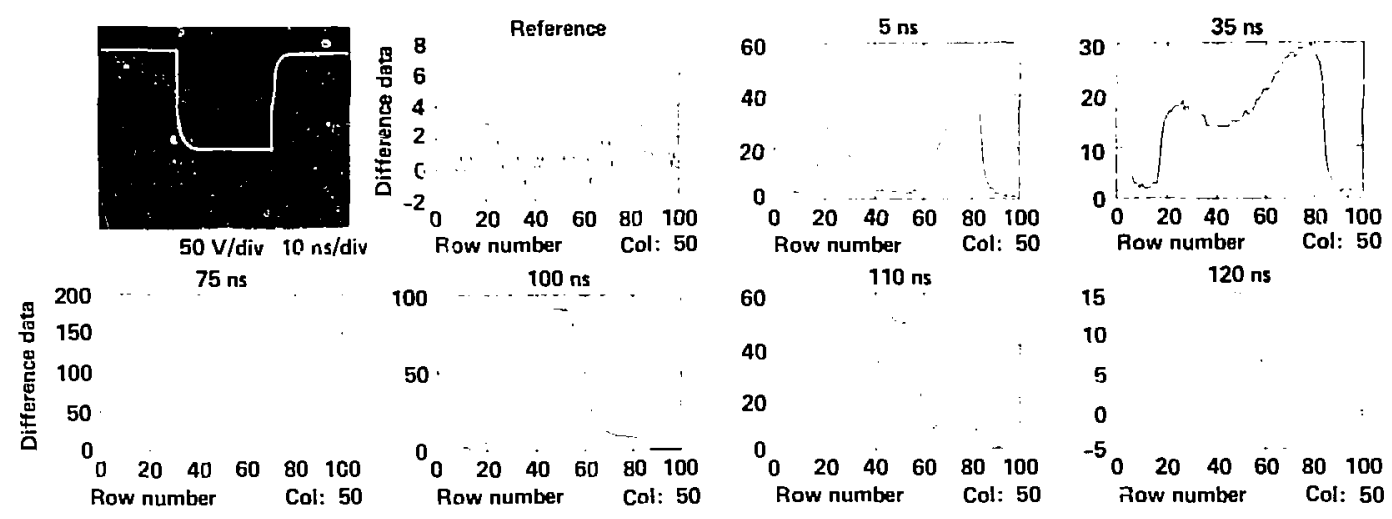

FIG. 5. Measured optical gate of an $18-\mathrm{mm}$ intensifier tube with a standard S20 photocathode. The first picture is the electrical pulse measured into $50 \Omega$. The electrical pulse had a peak voltage of $200 \mathrm{~V}$ with an FWHM of $38 \mathrm{~ns}$. The intensity profiles show the recorded inttensity across the diameter of the phosphor output of the intensifier tube as a function of time. Tote that the intensity of the input light was increased at $75 \mathrm{~ns}$ into the gate. As can be seen. the optical gate is much longer than the electrical driving pulse.

geometry. The highly resistive surface of the photocathode produces an "iris" effect as the electrical pulse propagates across its surface. The intensifier tube responded with a tum-on time of $40 \mathrm{~ns}$, a full-on time of $35 \mathrm{~ns}$. and a tum-off time of $45 \mathrm{~ns}$, thus producing a total optical gate of 120 ns. If the FWHM of the eleetrical pulse is decreased to $10 \mathrm{~ns}$, this intensifier tube never fully turns on in the center (Fig. 6).

When an ITT Model F41!1 18-mm intensifier tube that contains a metallic undercuating on the photocathode is used, the length of time for the electrical pulse to propagate from the edge to the center of the tube is greatly reduced. Figure 7 shows both the electrical pulse. which was coupled to the tube in a termination-type coupling, and the measured intensity profiles of an ITT F4lII intensifier tube with an undercoating of twice the standard thickness. When a $-150 \mathrm{~V}$ pulse with a 2-ns FWHM was applied. the intensifier tube responded with a turn-on time of 0.4 ns. a full-on time of 3.4 ns. and a turn-off time of 0.5 ns. giving a total optical gate of $4.3 \mathrm{~ns}$.

We crmpared the termination coupling with the microstrip transmission line coupling method. The same tuhe described in Fig. 7 in a termination coupling was also mounted in a 17-ohm microstripline pulser. The voltage pulse was adjusted to give approximately the same peak voltage and FWHM as were used in the termination geometry. Figure 8 shows the electrical pulse and measured intensity profile for the microstripline geometry. A total optical gate of $2.9 \mathrm{~ns}$ was obtained at a resolution of $40 \%$ CTF at $3 \mathrm{lp} / \mathrm{mm}$. This performance is compared with the 4.3-ns shutter obtained in the termination geometry. When the electrical pulse width was shortened to 1.2-ns FWHM with $50 \mathrm{~V}$ cross-over width of $1.5 \mathrm{~ns}$. the total optical gatc deereased to 1.9 ns as shown in Fig. 9.

We also tested a specially modified intensifier tube from Varo Corporation that had all of the photocathode except for a $3-\mathrm{mm}$ $x i 8-\mathrm{mm}$ strip masked off by a heavy meta] undercoating. The tube was mountid in a microstrip transmission line geometry, and the clectrical pulse was adjusted to give $-120 \mathrm{~V}$ with an FWHM of less than I ns when ineasured across the photocathode of the intensifier tube. This arrangement produced an optical shutter of $1.4 \mathrm{~ns}$ with a resolution of $3 \mathrm{lp} / \mathrm{mm}$ at $45 \%$ CTF.

When the back bias was raised to $110 \mathrm{~V}$, so that the forwarddriving bias to the tube was unly $10 \mathrm{~V}$, a total optical gate of less than 1 ns was obtained with a CTF of $38 \%$. Figure 10 shows the results of this test. No iris effect was observed, and the shading distortions were constant throughout the gate time.

The linearity of the camera system has been measured for optical gate widths from 4 to $250 \mathrm{~ns}$. In all cases, at wavelengths ranging from 380 to $700 \mathrm{~nm}$, the camera responded linearly over a range greater
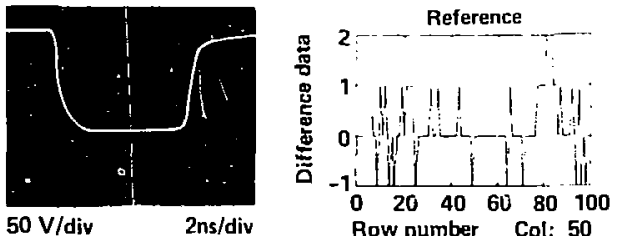

$50 \mathrm{~V} / \mathrm{div}$

2ns/div
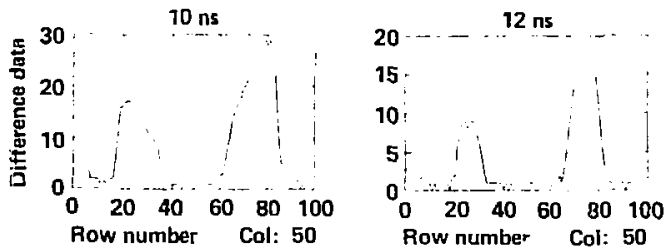
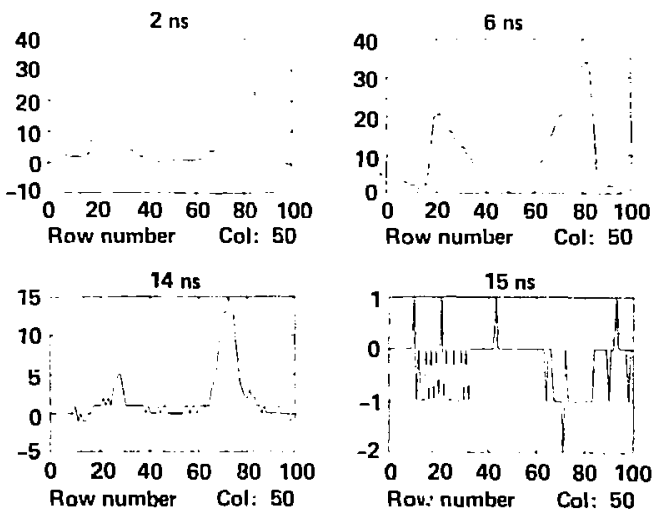

FIG. 6. Measured optical gate of an $18-\mathrm{mm}$ intensifice tube with a standard $\mathbf{S 2 0}$ photocathode in which the electrical pulse is not long enough to allow the intensifier tube to fully turn on in the center. The electrical pulse had a peak voltage of $190 \mathrm{~V}$ and an FWHM of 10 ns. In this case, the tuni-off proceeds from the center to the edges, whereas the turn-off of a fully turned-on tube proceeds from edge to center. 

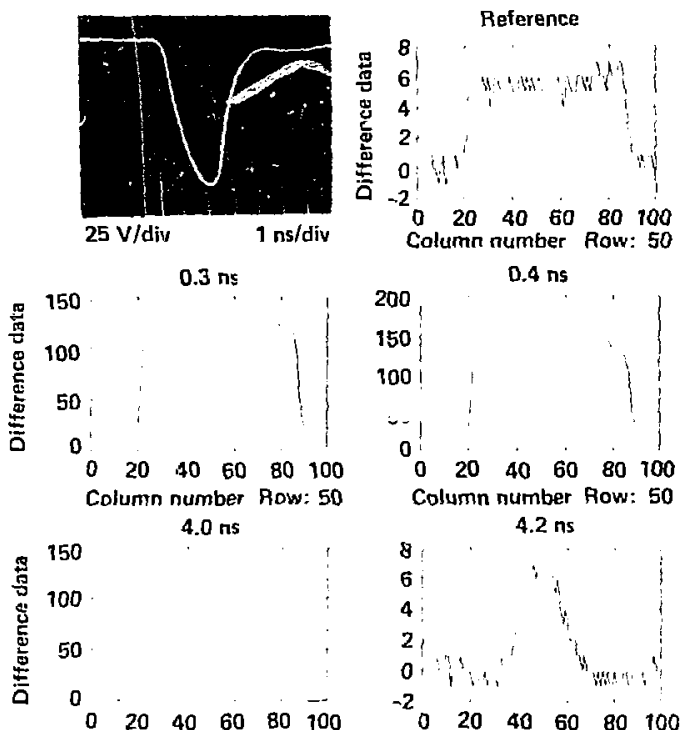

Column number

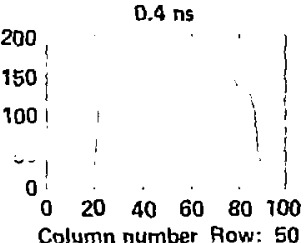

$4.2 \mathrm{~ns}$

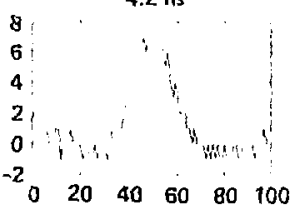

Column number Ruw: 50

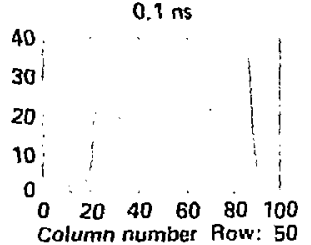

$1.0 \mathrm{~ns}$

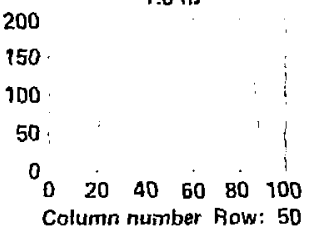

$4.3 \mathrm{~ns}$

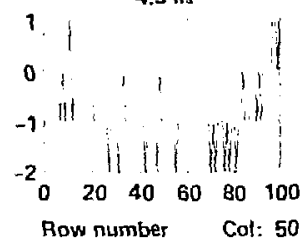

$0.2 \mathrm{~ns}$

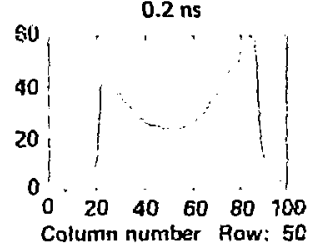

$3.0 \mathrm{~ns}$

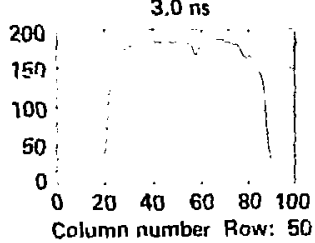

Resolution

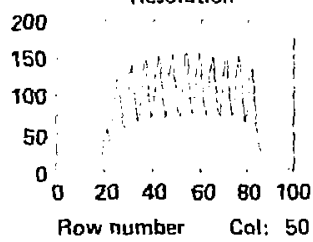

$3 \mathrm{la} / \mathrm{mm} 40 \%$ CTF

FlG. 7. Measured optical gate of an 18 -nm imensifier tube with a metallic layer under the photocathode. The "iris" effect is greatly reduced because of the lower surface resistance of the pholocathode.
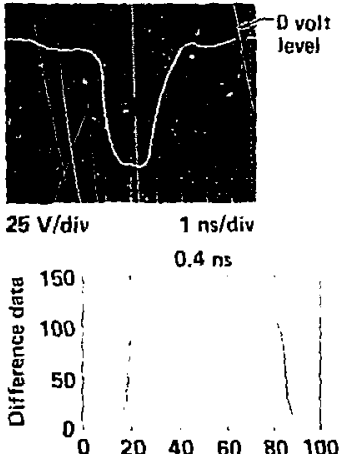

Column number Row: 50

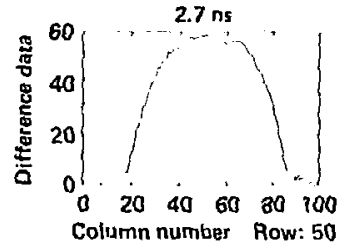

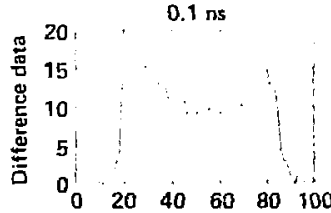

Column number Row: 50
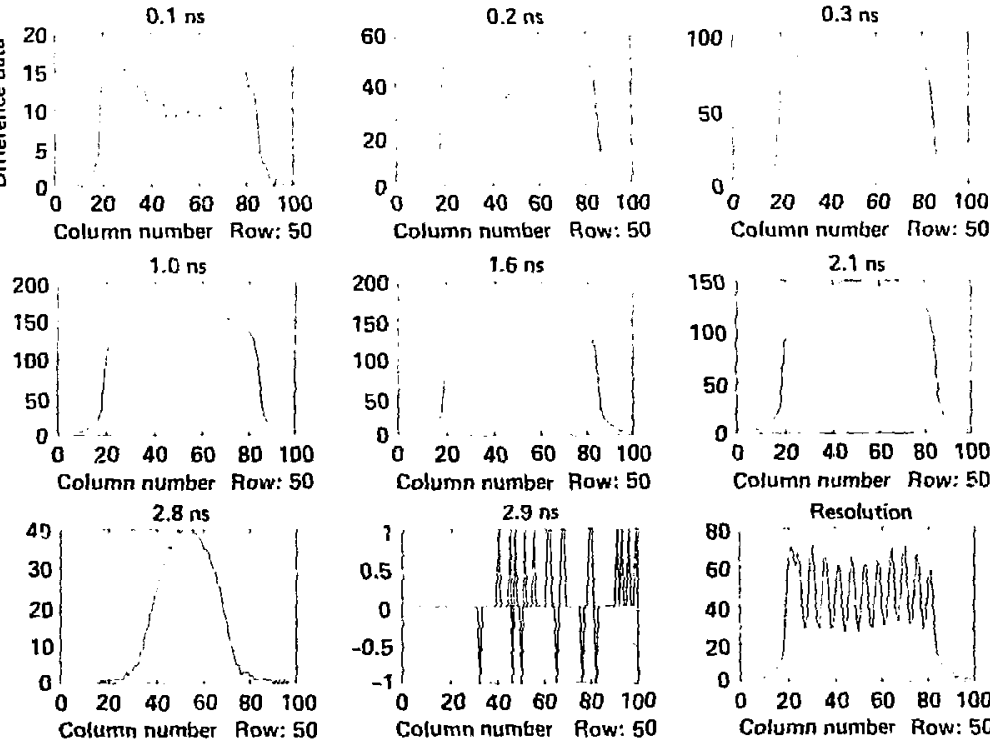

Column number Row: 50
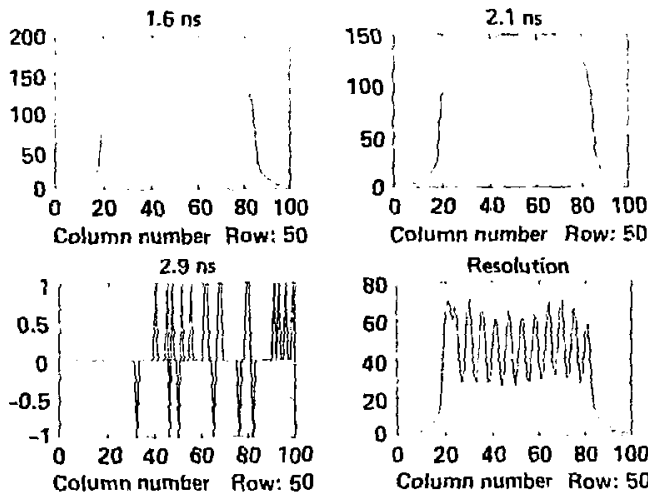

Column number Row: 50

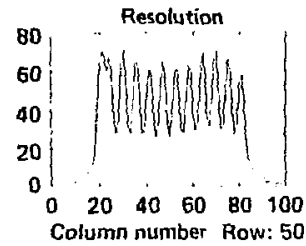

$3 \operatorname{lp} / \mathrm{mm} 40 \%$ CTF

FIG. 8. Measured optical gate when gating an intensifier tube in a microstrip transmission line amangement. The upper-lefl picture is the measured electrical pulse after passing over the photocathode. The ${ }^{+} w^{-4 s}$ that follow show the measured intensity profiles as a function of time. 


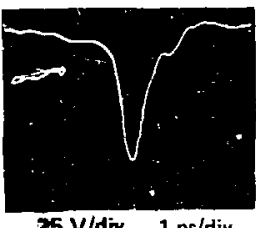

$25 \mathrm{~V} / \mathrm{div} 1 \mathrm{~ns} / \mathrm{div}$

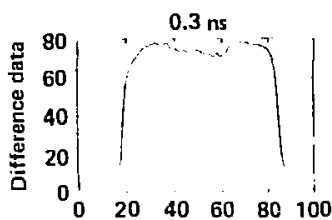

Column number Row: 50

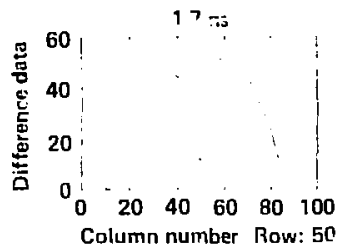

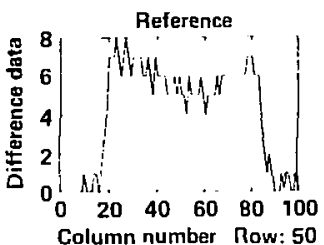

$0.8 \mathrm{~ns}$
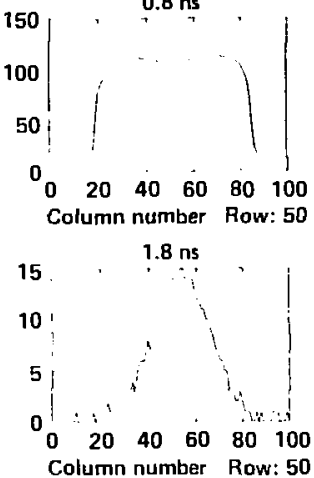

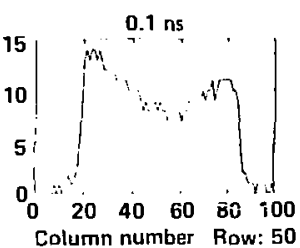

1.4 ns

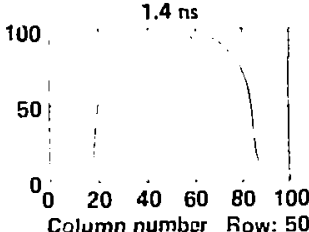

Column number Row: 50

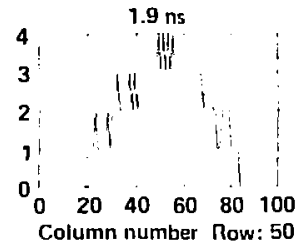

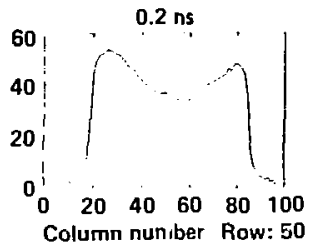

$1.6 \mathrm{~ns}$

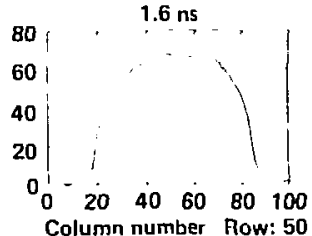

Resolution

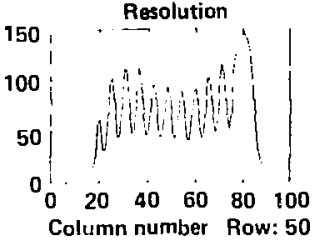

j $\mathrm{lp} / \mathrm{mm} 34 \%$ CTF

FIG. 9. Measured optical gate when gating an intensifier tube in a microstrip transmission line arrangernent with the electrical pulse adjustcd for the shonest possible pulse width.
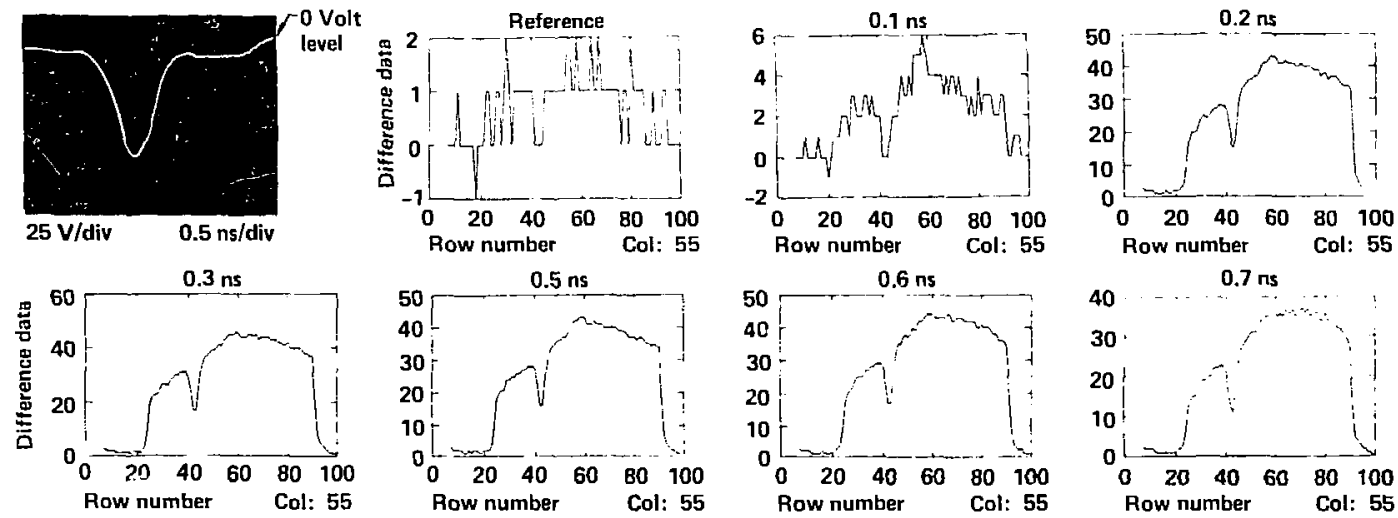

$0.7 \mathrm{~ns}$
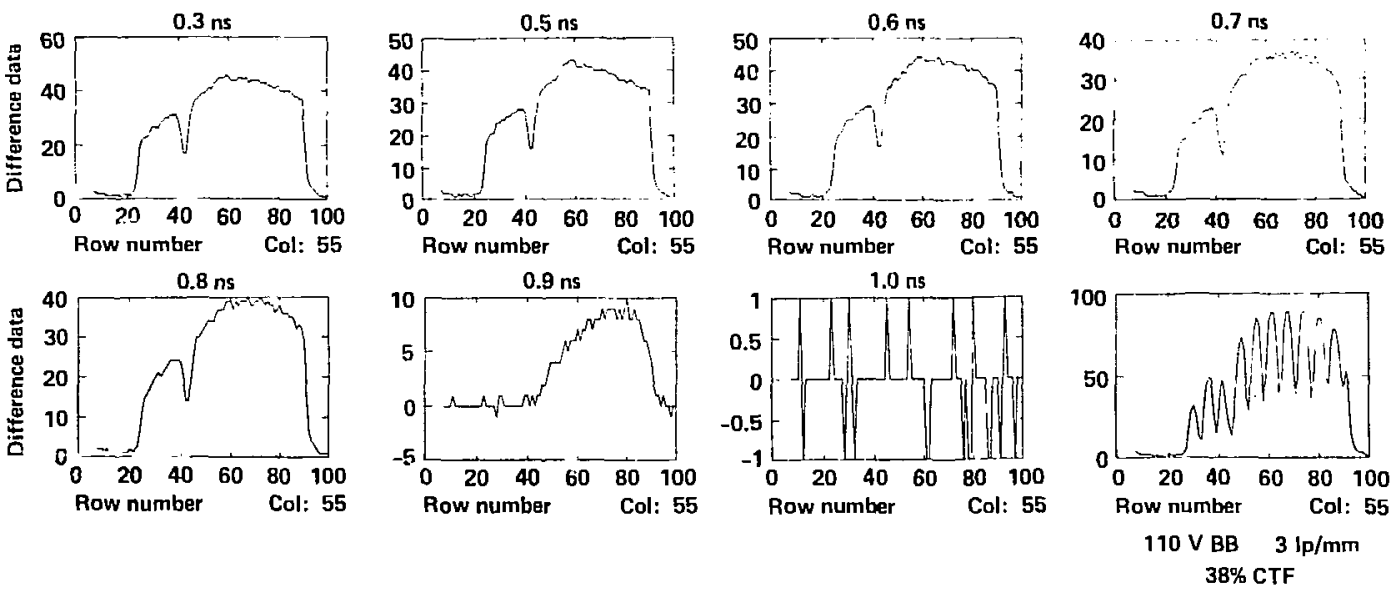

FIG. 10. Measured results of the gating performance of the modified intensifier tube when gating the intensifier tube in a microstrip transmission line geometry with a $110-\mathrm{V}$ back bias. The last picture shows the resolutinn of $31 \mathrm{p} / \mathrm{mm}$ at $38 \% \mathrm{CTF}$. 
than 200:1. This linearity indicates that the microchannel plate is not being depleted of its charge $*$ hen the intensifier tube is subjected to pulsed light. Figure 11 is a typjical plot of the measured linearity of the intensifier-camera combination. The system saturated at $73 \mathrm{pJ} / \mathrm{cm}^{2}$ into the photocathode.

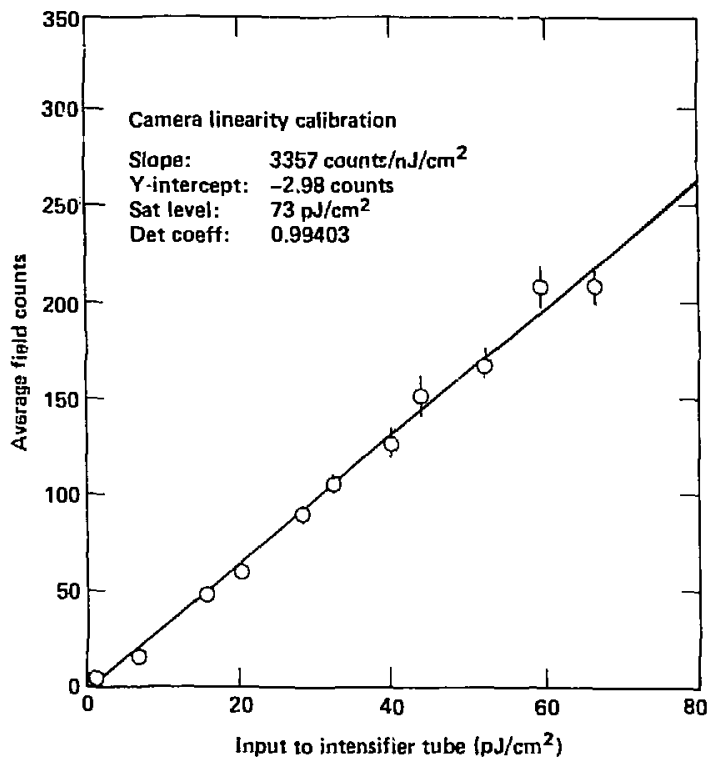

FIG. 11. Measured camera linearity of a Reticon $100 \times 100$ camera with a gated MCP intensifier tube fiber-optically coupled to the array. The camera saturated at $73 \mathrm{pJ} / \mathrm{cm}^{2}$. The output responded linearly with light input levels.

Uniformity vs time for a typical ITT F4111 tube is shown by the isometric plots in Fig. 12. The shading is believed to result from the irregularities in the resistivity of the photocathode undercoating. Both this nonuniformity and the gating time demonstrate that reducing the photocathode surface resistance is essential for fast gating. The surface resistance of $\mathrm{S} 20$ photocathodes has been measured to be approximately $10^{5} \mathrm{ohms} /$ square. $^{?}$

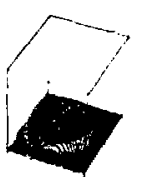

$0.1 \mathrm{~ns}$

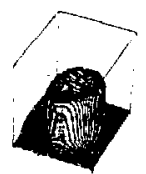

$0.3 \mathrm{~ns}$

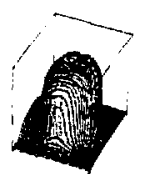

$2.0 \mathrm{~ns}$

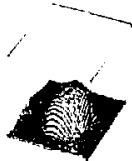

$4.0 \mathrm{~ns}$

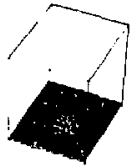

$4.1 \mathrm{~ns}$
FIG. 12. Isometric plots of a gated MCP intensifier tube vs time show very little nonuniformity vs time, except for the iris effect.

One of the best prospects for a fast optical gate is the thirdgeneration GaAs photocathode intensifier tubes. ${ }^{8-10}$ The resistivity of GaAs photocathodes has been reported to be less than 0.0 ! ohms/square, thus making it highly suited for fast gating. Preliminary results of gating these tubes coniritm that optical gates with a total duration of $\downarrow$ ns and no iris eflect are achievable.

\section{Downhole Streak Camera Applications}

SPARTEX (SPAtially Resolved Temperature EXperiment) is an experiment that measures the spatial dependence of temperature within a fusion cavity. SPARTEX uses the same "pinhole" imaging techniques as described above to image ionizing radiation onto a plas- tic fluorescer. The image from the fluor is focused onto a twodimensional image-disecting fiber-optic array that delivers the light signals to the input of a streak camera as a one-dimensional array, as shown in Fig. 13. The time dependence of each spatial point is recorded to an accuracy of $1 \mathrm{~ns}$ by a streak camera TV tamera system, which digitizes the data and transmits them to an uphole computer memory for storage. The thuor is positioned at least $25 \mathrm{~m}$ from the device so that time-of-flight techniques can be used to record the neutron speitral output from these resolved spatial poins. Each streak camera records tise neutron timie-of-flight spectrum for as many as 200 spatial points. From these data, a temperature is calculated for each point. The resultant profile of temperatures within the fusion casity gives information about device efriciency and implosion svmmetries.

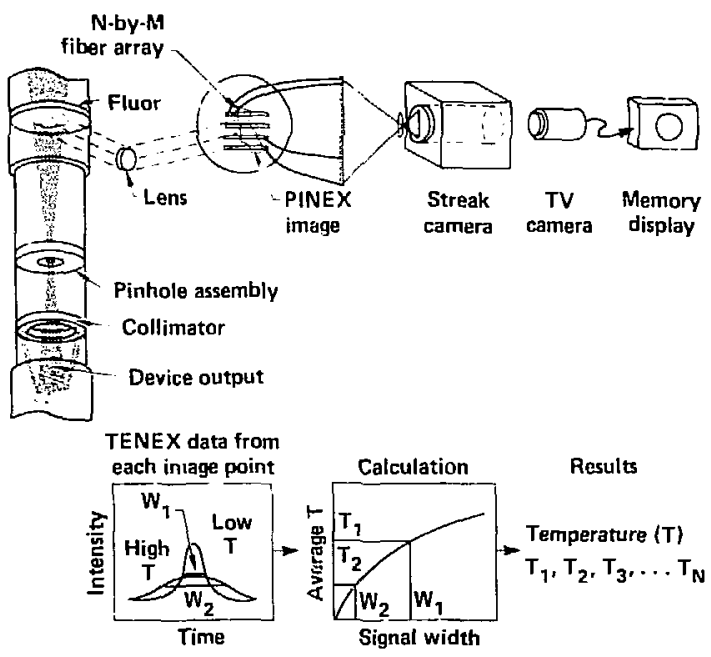

IIG. 13. SPARTEX (SPAtially Resolved Temperature EXperiment) uses the same pinhole imaging jechniques as PINEX. Light from the fluor is focus:d onto a special tiber optic image disector. which converts the two-dimensional image into a line image to be delivered to a streak camera. Each resulvable point records a neutron time-of-flight spectrum from which a local plasma temperature is calculated.

We are also developing a tomographic experiment to be used with streak camera recording. Special optics will compress the image to a line. The compression will be done from several angles through the image, and all of the compressions will be recorded on a streak camera. An algorithm will be used to reconstruct a two-dimensional image at many different times along the streak camera record. A slowmotion movie can then be rcconstructed from these images.

\section{Streak Camera Design ariu Performance}

The streak cameras used in these experiments are constructed around the RCA73435 AK and AJ series streak tubes, which have an S20 photocathode and a PII phosphor. The extraction grid in this tube is pulse-biased as a grating grid to improve the extinction ratio of the camera. The streak tube is fiber-optically coupled to an ITT $40-\mathrm{mm}$ microchannel plate image intensifier tube, which has an $\$ 20$ photucathode and a P20 phosphor. The image intensifier tube serves to adjust the sensitivity of the streak camera and to shift the output wavelength of the streak camera to better match with the Si response of the recording TV camera system. Details of this digital streak camera system are described in Table 2.

The dynamic range of the system is limited to approximately 200:I by the eight-bit digitizer of the TV camera. The spatial resolution of the system is limited by the Nyquist limit of the TV camera to 
Table 2. Digital streak camera components.

\begin{tabular}{ll}
\hline Streak tube & RCA 73435 AK\&AJ \\
Photocathode & S20 \\
Phosphor & P11 \\
& ITT MCP \\
Image intensifier & S20 \\
Photocathode & P20 \\
Phosphor & $10^{4}$ \\
Masimum gain & Fairchild CCD \\
Readout camera & 244 contiguous pixels \\
Spatial resolution & 380 pixels \\
Temporal resulution & 5 ms (3-ms future) \\
\hline
\end{tabular}

approximately 125 lines per camera width. Table 3 gives details on the streat: cumera performance separate from the readout system.

Table 3. Streak camera performance.

\begin{tabular}{ll}
\hline Spatial resolution & \\
Photocathode size & $38 \mathrm{~mm}$ \\
Resulvable lines & 300 \\
Temporal resolution & $<1 / 2 \%$ of sween \\
Dynamic range & $>100: 1 @ 65 \%$ CTF \\
Sensitivity & Detects single photoelectrons \\
& at phospnor \\
\hline
\end{tabular}

\section{Uphole Streak Camera Applications}

We are also developing a system that will allow these data to be recorded on uphule sireak cameras after being uransmited through up to $1 \mathrm{~km}$ of optical fiber. The technique employs wavelength multiplexing to allow sending several spatial points uver each optical fiber. The dita are demultiplexed uphole and presented to the photocathode of the streak camera as depicted in Fig. 14.

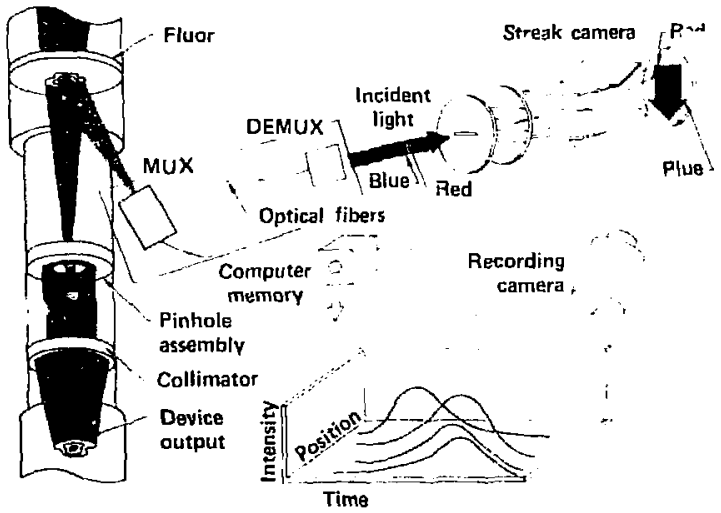

FIG. 14. Fiber Optic SPARTEX uses the same technology as SPARTEX except that the image disector consists of a wavelength multiplexer and several hundred meters of fibers. This allows the streak camera system to be used uphole. The downhole system is totally passive.

\section{Wavelength Multiplexing}

The wavelength multiplexer consists of a lens doublet with a wavelength dispersive element between the lenses. ${ }^{11-13}$ Thus, if the light from the object has a spectral distribution, a pcint of light at the object plane forms a spectral line at the image plane. as illustrated in
Fig. 15. The intensity information at the objective point is reproduced at every point along the spectral output line on the image plane. The length of the spectral output line is controlled by the amount of dispersion in the optical system.

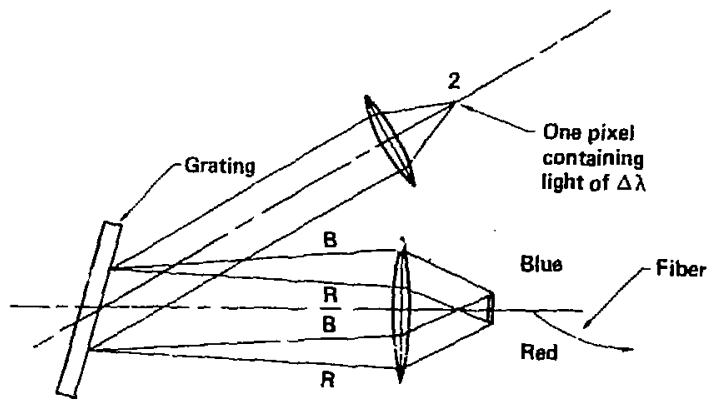

FIG. 15. A point at the objective plane is dispersed to a focused spectral line at the image plane of the multiplexer.

A dillraction grating was chosen as the dispersive element because it provides the greatest dispersion over the spectral range of the fluor. This system can also be used to encode a spatial line from the abjective plane. In this case, each point along the objective line is dispersed into a spectral line at the image plane. If the grating parameters are chosen properly, the "red" portion of the spectral output from one end of the objective line will coincide with the "blue" portion of the spectral output from the other end of the objective line. This is illustrated in Fig. 16, where the blue portion of the spectrum from point $\mathrm{Pl}$ coincides with the red portson of the spectrum from point P2. Thus. an optical fiber located at point $B$ collects information from each point along the objective line PIP2, by receiving each objective point as a different wavelength. Therefore, the optical fiber

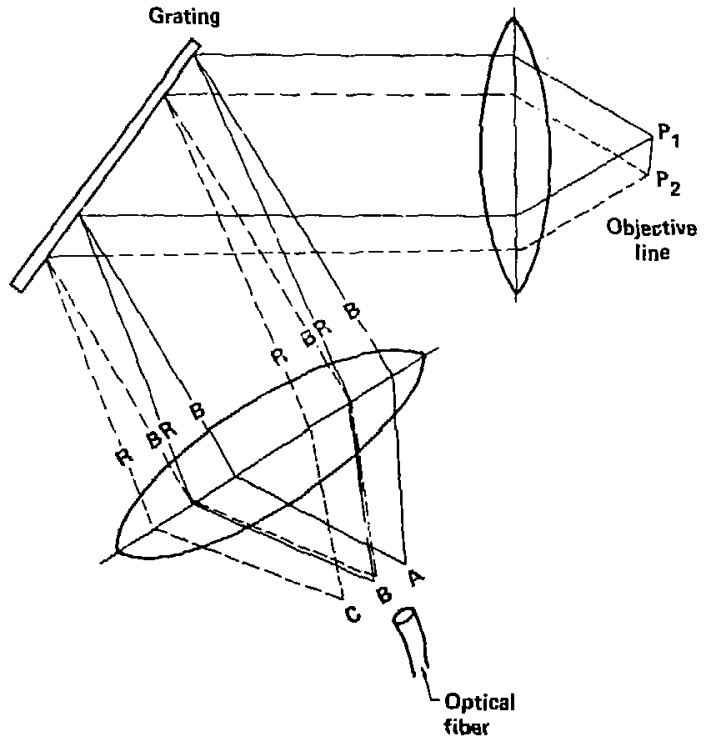

FIG. 16. Each point on a spatial line at the objective plane of the wavelength multiplexer is dispersed into a spectral line at the output. The blue portion of the spectrum from point $P_{2}$ is focused to the same position on the innge plane as the red portion of the spectrum from point $P_{1}$. 
carries spatial information about all points on the objective line P1P2 by encoding spatial position into wavelength, PIP.PIP., then injecting this information onto the fiber.

The spatial image can be denultiplexed from the optical fiber by using the same wavelength-dispersive optics in reverse. The final lineimage contains the same spatial information in the same position as the objective line but each position is also displayed as a different wavelength. Therefore, if accurate contrast information is desired, the resultant line-image must be corrected for the wavelength dependence of the sensor.

\section{Wavelength Multiplexer Design and Performance}

This svstem was designed to work in the spectral region from 500 to $600 \mathrm{~nm}$. Two $50-\mathrm{m}: n$ focal length $\mathrm{f} / 1.5$ lenses are used, and the gratings are blazed for $550 \mathrm{rm}$ with a spacing of 1200 groves/in. The dispersion is adjusted so that the spectral outpu: line from each point is 100 times longer than the $62-\mu$ ictive core diameter of the optical fibers that collect the light. That is, each spatiai sample is encoded into a !-nm-wide portion of the spectrum.

The transmission losses through the multiplexer ant demultiplexer are $3 \mathrm{db}$ each. Encoding each spatial point into $1 \mathrm{~nm}$ increases the loss by $20 \mathrm{db}$. Thus, there is an expected total of $26-\mathrm{db}$ loss for the multiplexing-demultiplexing system. The losses were measured to be $29 \mathrm{db}$. Typical optical fiber losses are $18 \mathrm{db} / \mathrm{km}$ at $550 \mathrm{~nm}$. Thus, the optical transport system is a $-47 \mathrm{db}$ loss over $1 \mathrm{~km}$ of optical fiher.

The limiting visual resolution at the output of the multiplexer is $15 \mathrm{lp} / \mathrm{mm}$ as determined by viewing the airy image with a telescope. This resolution is consistent with the $62-\mu$ optical fibe' diameter used to sample the dispersed light at the input end of the system. The optical fiber is effectively sampling the input as if it were a $62-\mu$ sliding aperture.

\section{System Tests}

The outputs from four optical fibers can be demultiplexed sequentially to form a line across the face of the streak tube. A $100-\mathrm{nm}$ wide bandpass filter centered at $550 \mathrm{~nm}$ is used in the demultiplexer so that the spatial out rout line from each fiber is limited to $6.2 \mathrm{~mm}$ (dispersion limit). At a resolution of $4 \mathrm{lp} / \mathrm{mm}$ on the streak camera photocathode, each input line is sampled 50 times.

The multiplexer system was tested by illuminating the input with a standard bar chart target, which has spatial bar patterns varying from 2 to $21 \mathrm{lp} / \mathrm{mm}$. At the $4-\mathrm{lp} / \mathrm{mm}$ resolution desired for the streak camera input, ine multiplexing system has a CTF of $90 \%$. Thus, the total system should give a resolution of $4 \mathrm{lp} / \mathrm{mm}$ at a CTF of approximately $60 \%$ over a linear dynamic range of $100: 1$.

A prototype system consisting of the multiplexer and streak camегa with film readout was assembled and tested. System performance is limited by the lignt intensity input to the wavelength multiplexer system. A power density of $103 \mathrm{~W} / \mathrm{cm}^{2}$ is required at the objecive plane of the multiplexer if the fult dynamic range of the streak camera is to be used. At this power density, the electron density at the phosphor of the streak tube gives 10\% statistics for a resolution of $4 \mathrm{lp} / \mathrm{mm}$. Statistics can be improved by reducing the gain of the image intensifier in the streak camera and increasing the input light (power density).

\section{Other Imaging Experiments}

Other time-dependent imaging experiments are being developed that will use the same hardware described above. Various forms of fiber optic image splitters are used in conjunction with streak cameras to measure material motion. This technique may eventually be combined with wavelength multiplexing for uphole recording.

We are also developing a time-dependent $x$-ray spectrometer that uses these same techniques. A special fluor is placed at the focal plane of an $x$-ray spectrometer. Light lrom this plane is fiscused into optical fibers and transmitted uphole to be recorded on a streak camera. The resultant data yield a time-dependent $x$-ray spectrum.

\section{Conclusions}

Time-dependent imaging is an important part of the diagnostics used by the Lawrence Livermore National Laboratory Test Program. Applications include material motion studies. time-of-flight background discrimination, and time-of-llight temperature measurements. Both fast framing camera and streak camera technolngies are employed.

Our comparison between termination-type coupling and microstrip transmission line coupling to intensifier tubes indicates that for optical shutters with a duratiun of a few nanoseconds, the microstrip transmission line geometry is superior to that of the simpler termination-type coupling. Also, the width of the electrical pulse from the microstrip transmission line pulser measured after passing over the photocathode agrees with the measured width of the optical gate, when the length of time taken for the iris is added to the pulse width at the $50 . \mathrm{V}$ level.

Optical shutters with exposure time of less than 1 ns have been achieved over limited regions of the photocathode, and exposure times of $1.9 \mathrm{~ns}$ have been achieved over the entire $18-\mathrm{mm}$ photocathode. We found it was essential to reduce the photocathode resistivity and to usc a microstrip transmission line coupling geometry. The GaAs photocathode shows the best prospect for obtaining an optical gate of $\mathrm{I}$ ns or less from an $18-\mathrm{mm}$ MCP intensifier tube. Preliminary results have shown that 1 -ns gating over the entire $18-\mathrm{mm}$ photocathodc surfacc is obtainable.

We have developed a digital streak camera for remote data recording. It is capable of recording 125 lines of data over a dynamic range of $200: 1$ and with a time resolution of $1 / 2 \%$ of the sweep time. We are also developing a wavelength multiplexing system that transmits spatially resolved data over $\mathrm{I} \mathrm{km}$ of optical fiber. The output of the multiplexer is focused on a streak camera for data recording. Prototype testing of the streak-camera-multiplexer system indicates a CTF of $60 \%$ at $4 \mathrm{lp} / \mathrm{mm}$, with a linear dynamic range of greater than 100:1.

\section{Acknowledgments}

I wish to thank the many people involved in this work at the Lawrence Livermore National Laboratory. Dave Kotecki performed most of the fast shutter work reported here. Hal Gates assembled and maintained the characterization laboratories we used. Lamar Olk eontributed pulser designs, streak camera design and testing, and many helpful suggestions on intensifier tube mounting. Tim Sammons helped with pulser design and fabrication. Bob Reedy was responsible for the optics design of the wavelength multiplexer. Barry Jaeoby contributed many helpful suggestions and calculations.

\section{References}

[1] G. L. Yales et al., "Overview of Pulsers for Nanosecond Gating of Image Shutter Tubes," The 15th Intemational Congress on High Speed Photography and Photonics, August 1982.

[2] Hewlett-Packard Application Note No. 918, "Pulse and Waveform Genc:ation with Step-Recovery Diodes."

[3] K. K. Li et al., "Optical Switches for Generation and Pulse Shaping of Ultrashor Electrical Pulses," SPIE, vol, 322, Picosecond Lasers and Applications, 1982.

[4] F. J. Leonberger and P. F. Moulton, "High Speed InP Optoelectronic Switch," Appl. Phys. Lett., vol. 35, no. 9, November 1, 1979.

[5] C. H. Lee, "Picosecond Optoelectronic Switching in GaAs," Appl. Phys. Lett.. vol. 30, no. 2. January 15, 1977. 
[6] G. Mourou and W. Knox, "High Power Switching with Picosecond Precision." Appl. Phys. Letl., vol. 35, no. 7, October 1, 1979.

[7] Measurements of 520 photocathode resistivity performed at the Lawrence Livermore National Laboratory by Ralph Kalibjian et al., private communication.

[8, I. P. Csorba. "Recent Advancements in the Field of Image Intensification: The Generation 3 Wafer Tube," Applied Optics. vol. 18, no. 14. July 15, 1979 .

[9] R. U. Martinelli and D. G. Fisher, "7 ue Application of Semiconductors with Negative Electron. ills. thy Surfaces to Electron Emission Devices," IEEE Proceed. ،gs, :ol. 62, no. 10, October 1974.
[10] D. G. Fisher et al., "Photomission Characteristics of Transmission-Mode Negative Electron AfTinity GaAs and (ln, Ga) As Vapor-Grown Structures," IEEE Transactions on Electron Devices, October 1974, pp. 641-649.

[11] A. W. Lohmann and H. W. Werlich, "Spatial Pulse Modulation," Applied Optics, vol. 10, no. 12, 1971, p. 2743.

[12] H. O. Bartelt. "Transmission of Two-Dimensional Images by Wavelength Multiplexing," Optical Communications, vol. 28, nu. 1. 1979. p. 45.

[13] A. A. Fricsem and V. Levy, "Parallel Image Transmission by a Single Optical Fiber," Optical Letters, vol. 2, no. 5, 1978, p. 133.

This work was done under the auspices of the U.S. Depanment of Energy by the Lawrence Livermore National Laboratory under Contract No. W-7405-Eng-48.

NMS/yh 